\title{
The Effect of Large Deflections of Joints on Foldable Miniature Robot Dynamics
}

\author{
Cem Karakadıoğlu ${ }^{1} \cdot$ Mohammad Askari $^{1}$ - Onur Özcan ${ }^{1}$ (1) \\ Received: 9 August 2019 / Accepted: 3 February 2020 / Published online: 29 February 2020 \\ (C) Springer Nature B.V. 2020
}

\begin{abstract}
In miniature robotics applications, compliant mechanisms are widely used because of their scalability. In addition, compliant mechanism architecture is compatible with the manufacturing methods used to fabricate small scale robots, such as "foldable robotics", where the size and the materials used allow much larger deflections. In this paper, the kinematics of compliant mechanisms used in miniature foldable robots are investigated with the assumption of nonlinear large deflections that occur at the flexure joints. The solution of the large beam deflection is acquired using elliptic integrals and is verified with finite element analysis and experiments on a simple small foldable leg linkage. The large deflection model takes joint strain energies into account and yields accurate estimations for load capacity of the mechanism as well as the necessary input torque for actuation of the leg. Therefore, the model presented can be used to estimate the load capacity of a miniature robot, as well as to select appropriate actuators. The work is also extended to estimate the compliant leg kinematics and rigid body dynamics of a foldable robot. The robot's large deflection simulation results are compared with experiments and a simplified rigid-link pin-joint kinematic model. Our results demonstrate the modeling accuracy of the two approaches and can be used by foldable robotics community when deciding on the strategy to choose for modeling their robots.
\end{abstract}

Keywords Foldable robots $\cdot$ Miniature robots $\cdot$ Legged robots $\cdot$ Robot dynamics

\section{Introduction}

Miniature robots have many exciting capabilities such as agile locomotion, high maneuverability, and ability to scale obstacles due to their small sizes and low weights. Such robots can be used to easily and silently access confined spaces, therefore theyare ideal for search and rescue operations under collapsed buildings and surveillance practices in hazardous environments. Despite these advantages and potential uses, the small-scale manufacturing challenges limit the widespread use of miniature robots. There are a few manufacturing techniques developed specifically for miniature robots, potentially the most influential one being the smart composite manufacturing (SCM) method [1]. SCM was one of the first methods developed for manufacturing miniature robots and is the ancestor to several other methods such as Printed Circuit MEMS (PC-MEMS) [2], Pop-up book Micro-Electro-

Onur Özcan

onurozcan@bilkent.edu.tr

1 Miniature Robotics Laboratory, Mechanical Engineering Department, Bilkent University, Ankara, Turkey
Mechanical System (Pop-Up MEMS) [3], and foldable robotics [4].

The aforementioned manufacturing techniques utilize different composite structures to manufacture mechanical structures. The laser type and the materials used, and the precision achieved during the manufacturing differ between members of this family of manufacturing methods, but the paradigm remains the same. The ball bearings and revolute joints created by conventional manufacturing methods become less efficient and more prone to wear as they are scaled down because surface forces, such as friction, start to dominate. In PCMEMS based techniques, the revolute joints are replaced with flexure joints, creating compliant mechanisms. Compliant mechanisms offer less movable parts and less assembly time and effort compared to traditional mechanisms that have rotating and sliding parts. On the other hand, the motion a compliant joint generates might have high repeatability if elastic deformations remain at specific limits. Because of these benefits, compliant joints find a common use in micro-structures, actuators and sensors, such as in MEMS, Scanning Probe Microscopy (SPM), Atomic Force Microscopy Scanning (AFM), and miniature robotic applications using SCM or descendant manufacturing methods [5-7]. 
Despite allowing easy scaling and manufacturing at the small scale, compliant mechanisms limit the design possibilities for miniature robot mechanisms. Since their motion comes from repeated bending of flexible parts, the joints in compliant mechanisms are subject to cyclic loading and fatigue failure. In addition, most foldable robot mechanisms are planar; therefore, mechanical stops are introduced to the system without pin joints. Hence, there is a limit to the deflection amount of a foldable robot joint [8]. For most compliant systems, the flexure joints experience small deflections. However, the materials used in miniature robots are often much more flexible, therefore the flexure joints can deflect $90^{\circ}$ or even higher.

Most of the kinematic models developed for foldable miniature robots consider the flexure joints as ideal pin joints or beams undergoing linear small deflections [9-12]. There are also several works, such as $[13,14]$, that model the joints in their walking or flying miniature robots using Howellâ $€^{\mathrm{TM}_{S}}$ pseudo-rigid-body model (PRBM) [8], where the flexure joints are treated as ideal revolute joints connected in parallel with torsional springs. These models are often preferred because they are easier to implement compared to nonlinear large deflection beam models. However, implementing small deflection or pure kinematic models for miniature foldable robots prevents accurate kinematic analysis of the compliant mechanism and affects the dynamic model of the robot altogether [15]. Most of the existing dynamic models of miniature mobile robots consider planar dynamics in sagittal- or horizontal-plane, such as [16], unlike some of the larger scale robots like Rhex [17], which describe the full body dynamics in space.

This paper presents a workflow for designing, modeling, and analyzing compliant mechanisms used in foldable miniature robots, whose joints undergo large deflections. Even though the study is based on a small foldable robot leg mechanism, the method used is not limited to miniature robotic applications. The flexure joints used in the foldable compliant mechanism are modeled as cantilever beams fixed from one end and subjected to combined end forces and moment loads, experiencing nonlinear large deflections. The solution of the large deflection model is acquired using elliptic integrals and the results are compared with finite element analysis (FEA) and experimental data for verification.

The methods for solving large deflection of beams and the effects of large deflections to compliant mechanism kinematics have been studied in literature before, and they are only summarized in this paper for the completeness of the analysis. The novel aspect of our work is using large deflection beam models for foldable miniature robot analysis to determine the effects of commonly overlooked large deflections on robot's payload capacity and robot dynamics. In this regard, the kinematic solutions for a simplified non-compliant model and the compliant large angle deflection model of the foldable leg mechanism of our quadruped robot, MinIAQ [6], are compared with each other.

The major contribution of this work is to implement nonlinear large deflection theory of cantilever beams to model the leg kinematics of mobile robots. This is applicable to origamiinspired and foldable robotics field where the flexure joint deflections may fall within the large angle regimes. Our contribution specifically is to compare the results acquired from both theories and the effects of the differences on robot dynamics. The large deflection model is significantly harder to implement but more accurate. This trade-off should be considered when modeling foldable miniature robots. A comparison is made to determine whether the gain in accuracy is worth the modeling efforts and higher computational cost.

This paper is organized as follows: Section 2 gives an insight into how compliant flexure mechanisms can be utilized in miniature foldable robotics by analyzing MinIAQ's leg mechanism design. Sections 3 and 4 formulate the EulerBernoulli linear small deflection and nonlinear large deflection beam theories, respectively, for the analysis of the robot's flexure joints. In Section 5, the presented theory is first verified with FEA and experiments on a single flexure joint. Next, the effect of payload on the verified compliant mechanism is investigated to determine the required motor torque. The developed methodology is then extended to analyze kinematics of MinIAQ's compliant leg mechanism. Lastly, in Section 6, the dynamic behavior of the robot with different kinematic solutions is investigated.

\section{Modeling of Compliant Mechanisms in Foldable Robots}

In legged robots, it is necessary to design legs such that the foot trajectory has long stride length, reasonable lift, and flat ground contact. However, such requirements are not easily attainable in small foldable robots as miniaturization and foldability aggravate the design difficulties. Miniature robot leg mechanisms are designed as compliant mechanisms to reduce frictional losses [2]. They are also actuated with minimal number of actuators to reduce costs and weight. In addition, foldability further limits the leg linkage selection to the ones for which unfolded crease patterns can be made. MinIAQ is a miniature foldable quadruped whose actuating mechanism is designed with these guidelines in mind [6]. The robot is approximately $10 \mathrm{~cm}$ long, weighs $23 \mathrm{~g}$, and has independent leg actuation as shown in Fig. 1. The flexure joints in its legs bend up to about $120^{\circ}$ at certain instances of a walking cycle.

Compliant mechanisms used in miniature foldable robots often consist of kinematic chains made from rigid links and flexure joints. Figure 2 illustrates the unfolded and folded configurations of MinIAQ's leg. The unfolded crease patternof the linkage, shown in Fig. 2(a), is first sketched in 


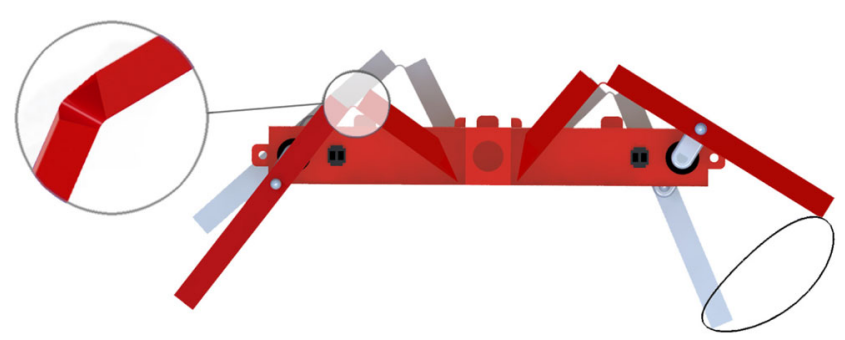

Fig. 1 Side view of MinIAQ. The locomotion mechanism consists of four compliant mechanisms individually actuated by a DC motor each. There are two flexure joints in each compliant mechanism. Inset shows two hollow triangular beams (rigid links) connected by a flexure joint. The black elliptic line represents the foot trajectory

2D. It is then cut from a thin flexible sheet under the laser and is folded successively over the perforations to yield the three dimensional shape (Fig. 2(b)). The folded triangular prismatic parts, which are locked in place with the help of fasteners, form the rigid links and the planar extensions between them are the flexure joints.

As the rotation of foldable flexure joints are limited, the crank link, which requires to make full rotation, must be made of a separate part. The crank should be attached to a DC motor, which is fixed within the body, and be connected to the compliant structure with a pin to form the closed chain fourbar mechanism (see Figs. 1 and 2(c)). Thus, the kinematic chain of foldable mechanisms in miniature robotics consist of several flexure joints, whose deflection amounts are limited, and

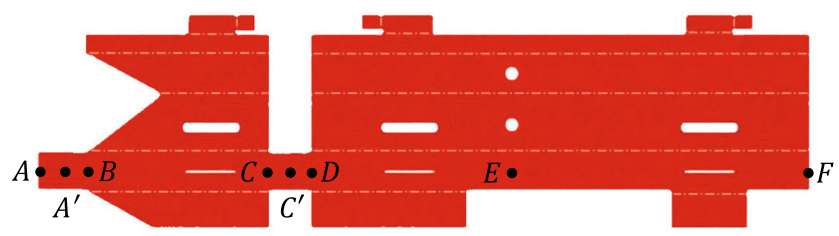

(a)

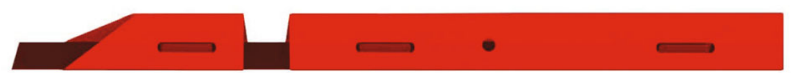

(b)

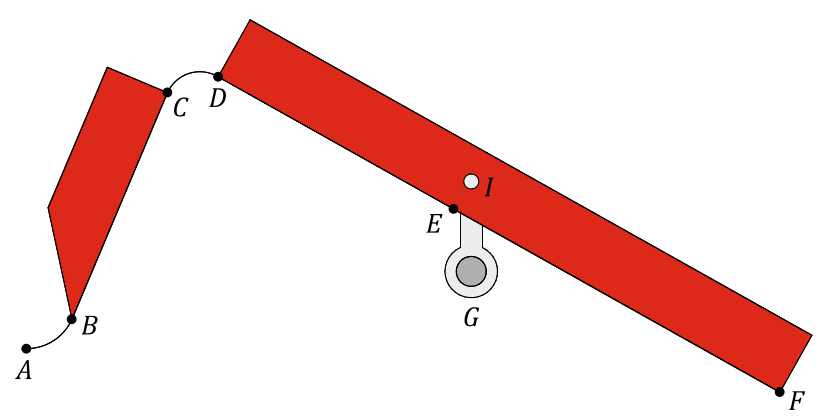

(c)

Fig. 2 Schematic of MinIAQ's foldable compliant leg mechanism. (a) The unfolded crease pattern. (b) The folded mechanism that is functionally equivalent to an open kinematic chain of two links and two flexure joints. (c) The closed chain fourbar linkage, corresponding to the assembled leg of the robot at least two pin joints: one connecting the motor to the cam and the other connecting the cam to the compliant mechanism.

Designing and modeling of origami robots require close examination of the correlations between unfolded and folded geometries. In designing origami legs, it is generally needed to transform a planar mechanism with ideal revolute joints into a foldable compliant mechanism. This requires careful design of the unfolded links geometries where small dimensional errors can result in an undesired leg linkage with poor foot trajectory. Similarly, in kinematic modeling of an existing leg, the modeling errors should be minimized. This must be done by examining both the folded and unfolded structures as well as taking all geometrical constraints into account when developing a leg model. The original version of MinIAQ consists of simple fourbar legs for the ease of planar design and assembly. Figure 3(a) shows a proper model of its compliant leg, where nodes $A$ and $G$ are fixed to the robot's body, $G I$ is the crank, $E I$ represents the small halved thickness of the coupler link, and the rest of the dimensions are measured with respect to the points shown along the joint line in Fig. 2(a).

Since the flexure joints in miniature robots undergo large deflections, the analysis of their compliant mechanisms requires solving complex nonlinear beam theories. This can be a major drawback in simulating a robot where leg kinematics is an integral part of a larger scale dynamic model and must be solved thousands of times for a few seconds of simulation. Consequently, for modeling purposes, it is very common to opt for solving a simplified model for kinematics for easier implementation and reduced runtime at the cost of accuracy. In an effort to address the validity of this assumption, we

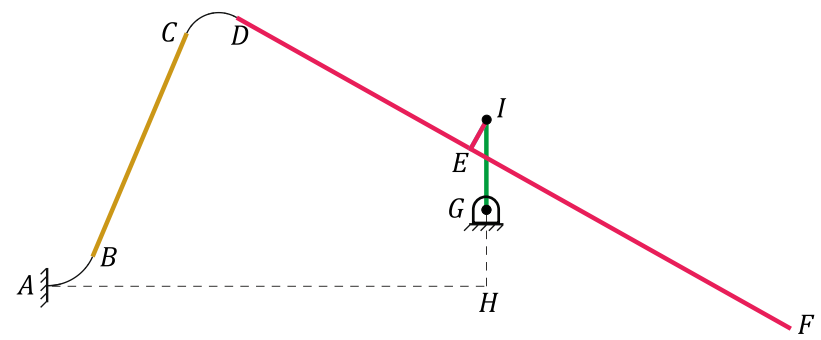

(a)

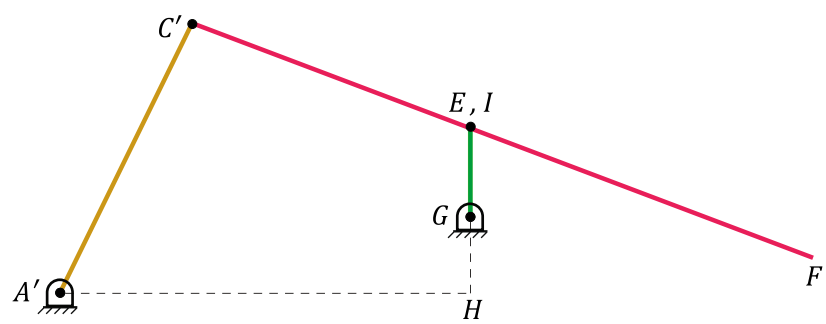

(b)

Fig. 3 (a) Schematic of MinIAQ's proper compliant leg model (the proposed model in this work), taking into account the flexure joints and actual geometric constraints. (b) A simplified fourbar model of the leg with pin joints and no correction for link shapes (the method generally used in the literature and in our previous works) 
examine the extent to which the dynamic model of MinIAQ is influenced by the leg kinematics simplification. In this regard, the results of dynamic simulation considering the accurate compliant leg model (Fig. 3(a)) are compared with those with a simplified fourbar leg (Fig. 3(b)). In the simplified fourbar, ideal pin joints are considered in place of flexure joints and the link shape geometric constraints are disregarded. Kinematic analysis solution of the fourbar mechanism is easy to obtain and is not addressed here for brevity. However, for the kinematic analysis of the compliant mechanism, necessary beam deflection theories are given and validated in the followingsections to properly model the flexure joints on MinIAQ.

\section{Linear Small Deflection Beam Theory}

From a mechanism modeling perspective, modeling of the motor-crank rotation is straightforward as the position of the crank can be easily determined for a given motor phase. On the other hand, modeling of the compliant structure is rather complicated. Deflection of the flexure joints can be modeled with linear deflection beam theory. In this regard, EulerBernoulli deflection equations of flexible cantilever beams should be first studied for combined end loads (see Fig. 4). According to Euler-Bernoulli elastic beam theory, bending moment, $M$, is proportional to its curvature, $k$, at any point along the beam. Hence, the curvature equation can be written as:

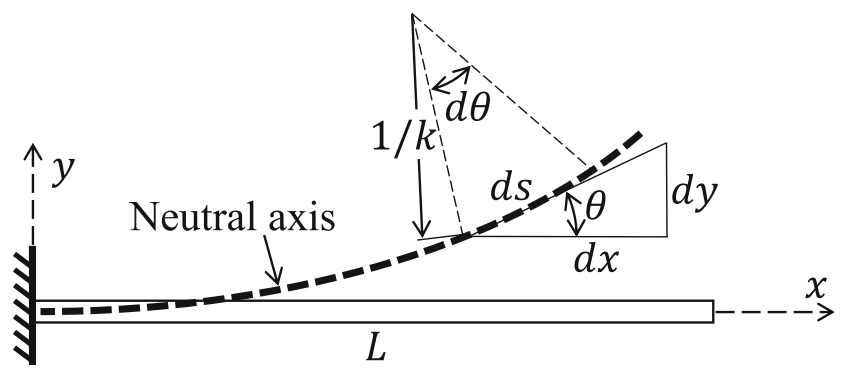

(a)

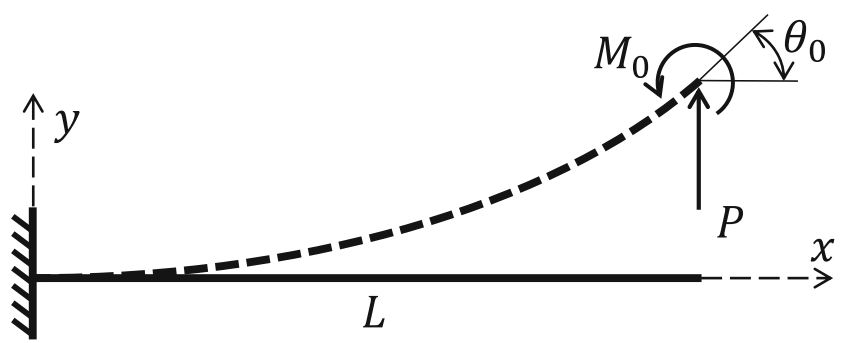

(b)

Fig. 4 (a) Euler-Bernoulli deflection of a flexure beam of uniform crosssection with length $L$, moment of inertia $I$, and Young's modulus $E$. (b) Combined force $P$ and moment $M_{0}$ loads acting on the free end of the cantilever beam with small deflections $k=\frac{d \theta}{d s}=\frac{M}{E I}=\frac{d^{2} y / d x^{2}}{\left[1+(d y / d x)^{2}\right]^{3 / 2}}$,

where $M$ is the bending moment, $d \theta / d s$ is the rate of change in angular deflection, $E$ denotes the Young's modulus of the material, and $I$ is the area moment of inertia of the cross-section. The rest of the terms in the equation are geometric parameters, as shown in Fig. 4. The linear moment-curvature relation is obtained for small deflection assumption by neglecting the $(d y / d x)^{2} \ll 1$ term in Eq. 1 .

The classical linear beam theory is originally developed for small or intermediate deflection of cantilever beams (up to about $10 \%$ of the length of a beam) [8]. This assumption may not very well account for the flexure joints in miniature robots which undergo large deflection angles. Nevertheless, the analysis of a foldable flexure joint with the linear beam theory is investigated as a proof of concept. The solution to Eq. 1 is well studied in the literature and only the final form of the solution is presented here for the sake of brevity [18]. The deflection and end slope of the cantilever beam for $x=L$ can be found by:

$y_{\max }=\frac{P L^{3}}{3 E I}+\frac{M_{0} L^{2}}{2 E I}$,

$\theta_{\max }=\frac{P L^{2}}{2 E I}+\frac{M_{0} L}{E I}$

and the moment at the fixed end of the beam (for $x=0$ ) is given by:

$M_{\text {max }}=P L+M_{0}$.

\section{Nonlinear Large Deflection Beam Theory}

The nonlinear large angle deflection theory is more appropriate for predicting large deformations in cantilever beams. In general, nonlinearities from large strains must be taken into account if the strain is large enough to cause significant changes in the geometry, such as in area or thickness of the beam [8]. However, these cases are not considered under this work because such geometrical changes are not dominant in miniature foldable robots where the end loads are small and the materials used are highly flexible.

Various methods exist in the literature to analytically solve for nonlinear large angle deflections of beams, such as, elliptic integral solutions [8, 19-28], pseudo-rigid-body model (PRBM) methods [8, 29-37], chained beam constraint model [8, 38-40], Adomian decomposition [19, 41] and circle-arc method [42]. Elliptic integral solutions are considered to be the most accurate ones in predicting large deflections as long as the end slope and load parameters are known. In the 
absence of these parameters, the solution should be found using numerical techniques such as finite element analysis (FEA). However, elliptic integral solutions converge very fast compared to finite element methods. Fast solution convergence is beneficial in kinematic or dynamic problems, such as in optimization of a compliant mechanism or in simulating full body dynamics.

Elliptic integral solution was first derived by Bisshopp and Drucker for a vertical force acting on the free end of a cantilever beam [20]. The solution was improved by Frish-Ray et al. for various loading cases [8, 22, 23, 25, 43]. The loading modes, leading to the formation of an inflection point, are modeled by Shoup et al. [8, 21, 24, 26, 27]. Beam with combined end forces and moment loads acting on its free end (see Fig. 5) is the hardest to derive elliptic equations for, especially when combined end forces and moments are acting in opposite directions, in which an inflection point may occur. A comprehensive solution for such loading case is derived by Zhang and Chen [28] and only a brief summary of the solution process is presented here.

The generalized curvature equation for large angle deflection of a cantilever beam subject to combined end loads is given by:

$$
\frac{d \theta}{d s}= \pm \sqrt{\frac{2 P}{E I}\left(\sin \theta_{0}-n \cos \theta_{0}-\sin \theta+n \cos \theta+\frac{M_{0}^{2}}{2 P E I}\right)},
$$

where $E I$ is the flexural rigidity of the beam, $\theta_{0}$ denotes the deflected angle of the beam end, $P$ and $n P$ are the components of the known end force $\eta P$, and the rest of the terms are shown on Fig. 5. Note that the sign in front of Eq. 5 is selected as positive for a concave upward curvature or as negative for a convex downward solution. This equation is often simplified to the following form:

$\frac{d \theta}{d s}= \pm \sqrt{2} \frac{\alpha}{L} \sqrt{\lambda-\sin \theta+n \cos \theta}$,

using a dimensionless load coefficient $(\lambda)$, a dimensionless force index $(\alpha)$, and a load ratio $(\kappa)$ defined by (see [8]):

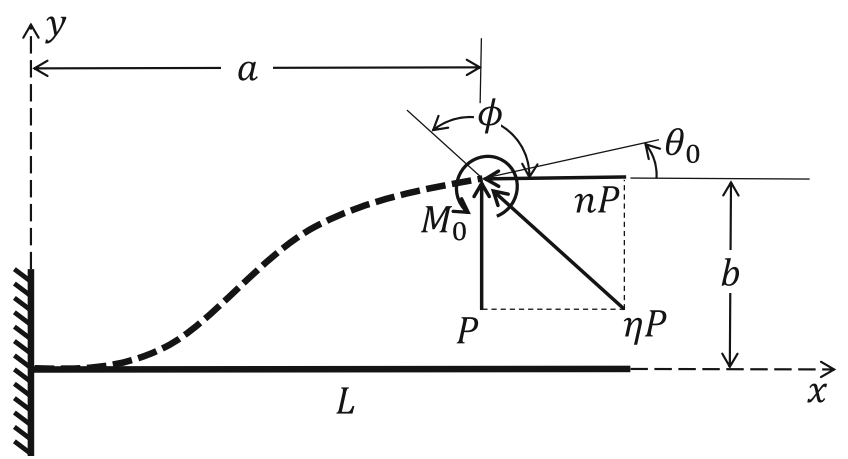

Fig. 5 Combined forces and moment loads acting to the free end of cantilever beam with large deflections
$\alpha^{2}=\frac{P L^{2}}{E I}$,

$\kappa=\frac{M_{0}^{2}}{2 P E I}$,

$\lambda=\sin \theta_{0}-n \cos \theta_{0}+\kappa$.

The solution to the curvature equation is expressed by:

$\alpha= \pm \frac{1}{\sqrt{2}} \int_{0}^{\theta_{0}} \frac{d \theta}{\sqrt{\lambda-\sin \theta+n \cos \theta}}$,

$b= \pm \frac{L}{\sqrt{2} \alpha} \int_{0}^{\theta_{0}} \frac{\sin \theta d \theta}{\sqrt{\lambda-\sin \theta+n \cos \theta}}$,

$a= \pm \frac{L}{\sqrt{2} \alpha} \int_{0}^{\theta_{0}} \frac{\cos \theta d \theta}{\sqrt{\lambda-\sin \theta+n \cos \theta}}$.

where $a$ and $b$ are the horizontal and vertical deflection of the beam, respectively.

Equations (10) to (12) are applicable when there is no inflection point on the flexure beam. Cantilever beam with combined loads may or may not have an inflection point depending on the end moment and the end force directions [24]. If the bending caused by the end moment is in the opposite direction of the bending caused by the end load, an inflection point will occur. On an inflection point, $k=d \theta / d s=0$ and $M=0$. In such a case, Eqs. (10) to (12) must be rewritten as follows:

$\alpha=\frac{(-1)^{m} S_{m}}{\sqrt{2}} \sum_{j=0}^{m}(-1)^{j} \int_{\widehat{\theta}_{j}}^{\widehat{\theta}_{j+1}} \frac{d \theta}{\sqrt{\lambda-\sin \theta+n \cos \theta}}$,

$a=\frac{(-1)^{m} S_{m} L}{\sqrt{2} \alpha} \sum_{j=0}^{m}(-1)^{j} \int_{\hat{\theta}_{j}}^{\hat{\theta}_{j+1}} \frac{\cos \theta d \theta}{\sqrt{\lambda-\sin \theta+n \cos \theta}}$,

$b=\frac{(-1)^{m} S_{m} L}{\sqrt{2} \alpha} \sum_{j=0}^{m}(-1)^{j} \int_{\hat{\theta}_{j}}^{\hat{\theta}_{j+1}} \frac{\sin \theta d \theta}{\sqrt{\lambda-\sin \theta+n \cos \theta}}$,

where $m$ is the number of inflection points, $\widehat{\theta}$ is the deflection angle at an inflection point, $j$ specifies the $j^{\text {th }}$ inflection point starting from the fixed end, and $S_{m}$ is the sign of the end moment load, $M_{0}$, which is positive if $M_{0}$ is counterclockwise and negative if clockwise. These equations can be solved with the help of elliptic equations of the first and the second kind (see [28] for detailed derivation).

\section{Results and Discussion}

\subsection{Verification of the Nonlinear Large Deflection Model with a Single Flexure Joint}

In order to demonstrate the difference between the small and large deflection solutions and verify the model presented in 


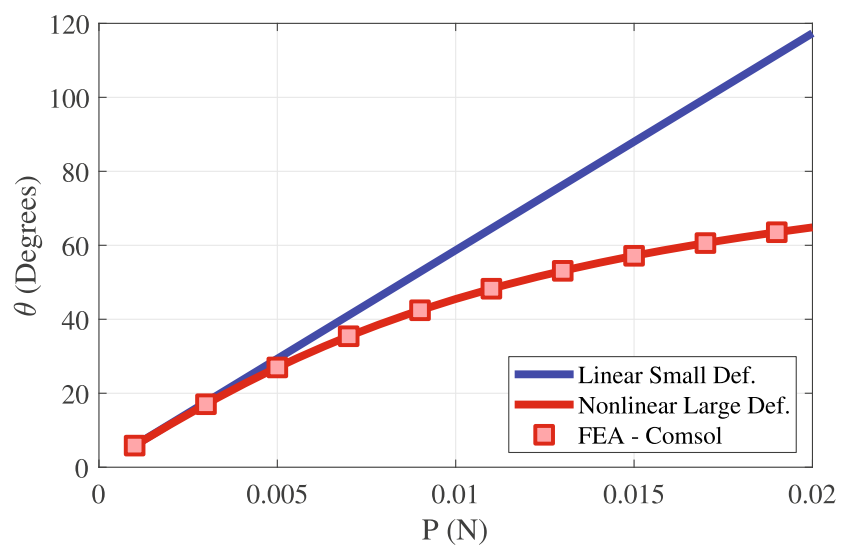

Fig. 6 End slope of a cantilever beam (flexure joint), $\theta$, versus vertical end load, $P$, for small and large deflection models. Joint parameters: $L=2 \mathrm{~mm}, b=6 \mathrm{~mm}, t=25 \mu \mathrm{m}, M_{0}=0 \mathrm{~N} \cdot \mathrm{m}$ and $P=0.001 \mathrm{~N}$ to $0.02 \mathrm{~N}$. Material properties of Kapton is used, which is a commonly utilized material for foldable robot fabrication

Section 4, a simple cantilever beam, subject to a vertical force only, is modeled in MATLAB using the linear beam theory and elliptic integral solution of the nonlinear large deflection model. In addition, a FEA is also conducted on the same beam in COMSOL environment. The comparison of these results are presented in Fig. 6.

When a compliant joint experiences large deflections, linear model formulations given in Section 3 yield inaccurate results because of the geometric nonlinearities arising from large deflections. Such cases occur often in foldable miniature robot applications and therefore, large angle deflection models should be used to model the mechanisms. As can also be seen in Fig. 6, beyond almost $30^{\circ}$ of deflection, the behavior becomes nonlinear and the linearbeam theory prediction becomes inaccurate.

Besides using FEA to verify the results, a single-flexurejoint mechanism is designed to compare the large deflection theory with experiments. Theoretical and experimental results of the vertical force acting on the compliant joint under different deflection angles are compared. The setup used for the experiments can be seen in Fig. 7(a). The rigid links of the mechanism are made of a sandwich of cardboard-acrylic adhesive-Kapton-acrylic adhesive-cardboard, whereas the joints are made out of bare Kapton (polyimide film). The vertical force acting on the compliant joint is measured by a force sensor connected at the base as the servo motor rotates the crank. Note that unlike Fig. 6, where the beam is assumed to be under a vertical force only, the flexure joint in the test setup is subject to combined force and moment loads.

In order to simulate the system, the mechanism's kinematic model should first be developed. A skeleton diagram of the mechanism is shown in Fig. 7(b). In this mechanism, $A C$ is a compliant joint while $A E, B C, C D, \mathrm{BD}$ and $B E$ are straight links and their lengths are denoted by $L_{i}$, where $i$ refers to the link name. $A E$ is the fixed motor arm connected to the servo whose shaft is coincident with point $A$ but is placed behind the mechanism and is not visible in Fig. 7(a). The input motor angle is denoted by $\psi$ and $\theta_{0}$ is the deflected angle of the compliant joint end. $L_{B C}$ is an unknown variable as $B$ is an auxiliary point along $C D$, which indicates the varying position of the slider joint as the motor turns. Also note that point $D$ is the tip of the arm to which a payload can be attached (not done in this experiment, $D_{x}=D_{y}=0$ ). The known parameters of the setup arepresented in Table 1, with the length, width, thickness, and Young's modulus of the compliant joint denoted by $L, w, t$, and $E$, respectively.

The kinematic position analysis can be expressed by the loop closure equations as follows:

$Q_{x}+L_{B C} \cos \theta_{0}+L_{B E} \cos \left(\theta_{0}+\frac{\pi}{2}\right)-L_{A E} \cos \psi=0$,

$Q_{y}+L_{B C} \sin \theta_{0}+L_{B E} \sin \left(\theta_{0}+\frac{\pi}{2}\right)-L_{A E} \sin \psi=0$,

in which the joint coordinates $\left(Q_{x}, Q_{y}\right), L_{B C}$, and $\theta_{0}$ are unknown. As deflection of beams are dependent on the applied force and moment, the combined end loads at the compliant joint must be determined. Figure 8 shows the free body diagrams of the links in the test setup. Thus, a set of equations
Fig. 7 (a) A single joint compliant mechanism test setup, used in experiments to verify large deflection analysis. (b) Skeleton diagram of the mechanism showing the trajectory path of the tip

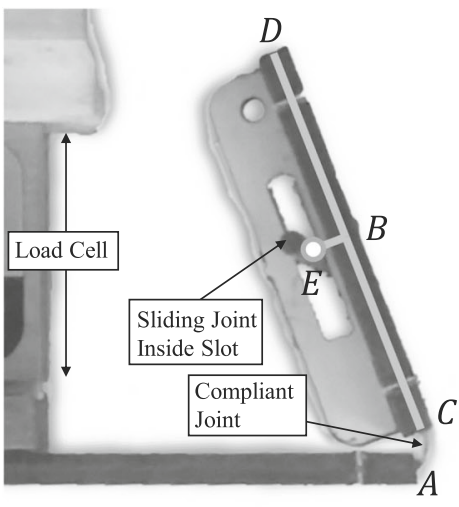

(a)

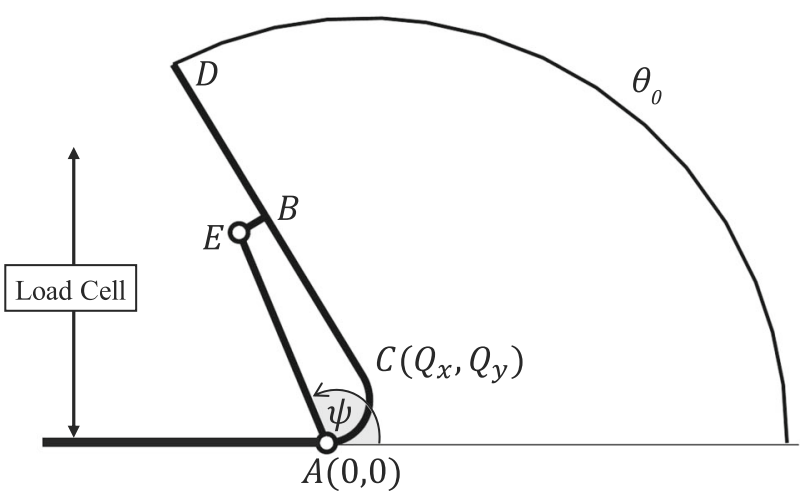

(b) 
Table 1 The fixed parameters of the compliant joint and links in the test setup

\begin{tabular}{lllllll}
\hline$L_{A E}(\mathrm{~mm})$ & $L_{C D}(\mathrm{~mm})$ & $L_{B E}(\mathrm{~mm})$ & $L(\mathrm{~mm})$ & $w(\mathrm{~mm})$ & $t(\mu \mathrm{m})$ & $E(\mathrm{GPa})$ \\
\hline 15.0 & 26.5 & 2.0 & 4.0 & 6.0 & 125 & 2.5 \\
\hline
\end{tabular}

comes from static equilibrium of link $C D$ :

$\sum F_{x} \Rightarrow D_{x}+F_{B} \cos \left(\theta_{0}+\frac{\pi}{2}\right)+n P=0$,

$\sum F_{y} \Rightarrow D_{y}+F_{B} \sin \left(\theta_{0}+\frac{\pi}{2}\right)-P-m g=0$,

$\sum M_{C} \Rightarrow F_{B} L_{B C}+L_{C D}\left[D_{y} \cos \theta_{0}-D_{x} \sin \theta_{0}\right]$

$-m g \cos \theta_{0} L_{C D} / 2-M_{0}=0$,

where $m g$ is the weight of the arm and the new unknown variables are $F_{B}, P, n P$, and $M_{0}$. Using the schematic in Fig. 8(d), the following geometric constraints can be written:

$L_{A C}^{2}=Q_{x}^{2}+Q_{y}^{2}$,

$L_{C E}^{2}=L_{B E}^{2}+L_{B C}^{2}$

$L_{C E}^{2}=L_{A E}^{2}+L_{A C}^{2}-2 L_{A E} L_{A C} \cos (\psi-\beta)$,

$\beta=\operatorname{atan} 2\left(Q_{y}, Q_{x}\right)$,

which yield an extra equation in terms of the unknowns:

$$
\begin{aligned}
& L_{A E}^{2}-L_{B E}^{2}-L_{B C}^{2}+\left(Q_{x}^{2}+Q_{y}^{2}\right) \\
& \quad-2 L_{A E} \sqrt{Q_{x}^{2}+Q_{y}^{2}} \cos \left(\psi-\operatorname{atan} 2\left(Q_{y}, Q_{x}\right)\right)=0 .
\end{aligned}
$$

The system of Eqs. (16) to (21) consists of six nonlinear equations and eight unknown variables: $\theta_{0}, Q_{x}, Q_{y}, L_{B C}, F_{B}, P$, $n P$, and $M_{0}$. At least two more equations are needed in order to solve for the unknowns. These equations come from modeling the bending of the compliant joint using the linear or nonlinear deflection beam theories presented in Sections 3 and 4. If the joint is modeled with the linear small deflection theory, the extra equations are:
$Q_{y}-\frac{P L^{3}}{3 E I}-\frac{M_{0} L^{2}}{2 E I}=0$,

$\theta_{0}-\frac{P L^{2}}{2 E I}-\frac{M_{0} L}{E I}=0$,

whereas, if it is modeled with nonlinear large deflection beam theory, the following equations must be used instead:

$$
\begin{aligned}
& \alpha-\frac{(-1)^{m} S_{m}}{\sqrt{2}} \sum_{j=0}^{m}(-1)^{j} \int_{\hat{\theta}_{j}}^{\hat{\theta}_{j+1}} \frac{d \theta}{\sqrt{\lambda-\sin \theta+n \cos \theta}}=0, \\
& Q_{x}-\frac{(-1)^{m} S_{m} L}{\sqrt{2} \alpha} \sum_{j=0}^{m}(-1)^{j} \int_{\hat{\theta}_{j}}^{\hat{\theta}_{j+1}} \frac{\cos \theta d \theta}{\sqrt{\lambda-\sin \theta+n \cos \theta}} \\
& =0, \\
& Q_{y}-\frac{(-1)^{m} S_{m} L}{\sqrt{2} \alpha} \sum_{j=0}^{m}(-1)^{j} \int_{\hat{\theta}_{j}}^{\hat{\theta}_{j+1}} \frac{\sin \theta d \theta}{\sqrt{\lambda-\sin \theta+n \cos \theta}}=0,
\end{aligned}
$$

where $\lambda=\sin \theta_{0}-n \cos \theta_{0}+M_{0}^{2} / 2 P E I, \quad I=w t^{3} / 12$, $\alpha=L \sqrt{P / E I}$, and the rest of the parameters are as defined in Section 4. When the joints are modeled as nonlinear beams with large deflections, the system of equations become over constrained and a nonlinear trust-region based solver is used to obtain a solution. Note that for the linear case, no extra constraint is added to $Q_{x}$, which makes the horizontal length of the beam, or in other words the total length of the bent beam, a free parameter. This is indeed against the original assumption of the linear deflection theory, which takes the horizontal length to always remain equal to the total beam length, L, (see Fig. 4). However, such an assumption creates a singularity at $90^{\circ}$ deflection, and thus we do not impose a
Fig. 8 Free body diagrams of (a) the compliant joint, (b) the crank connected to the motor, $A E$, and (c) the rigid arm link, $C D$. (d) A schematic of the single leg compliant mechanism setup with dashed lines to clarify geometric relations

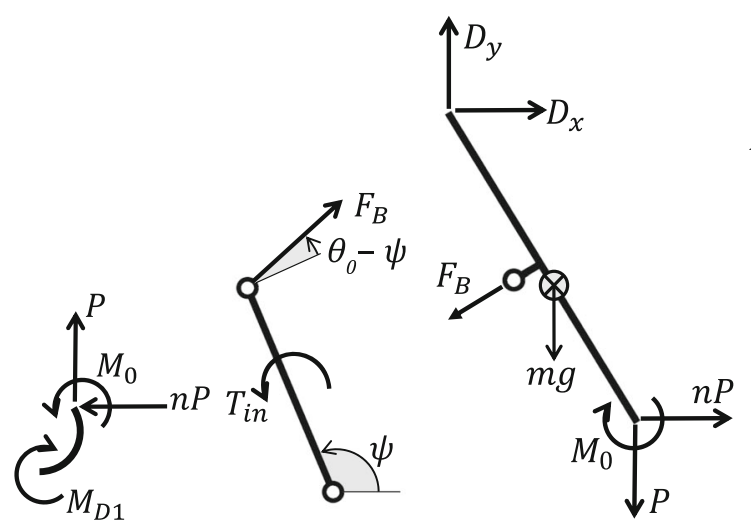

(a) (b)

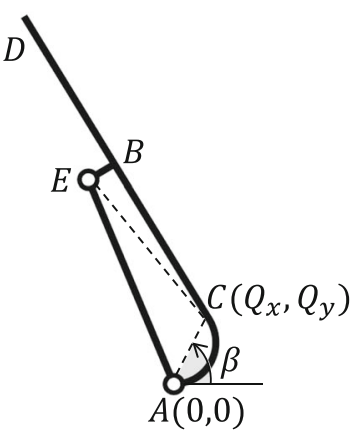

(d) 


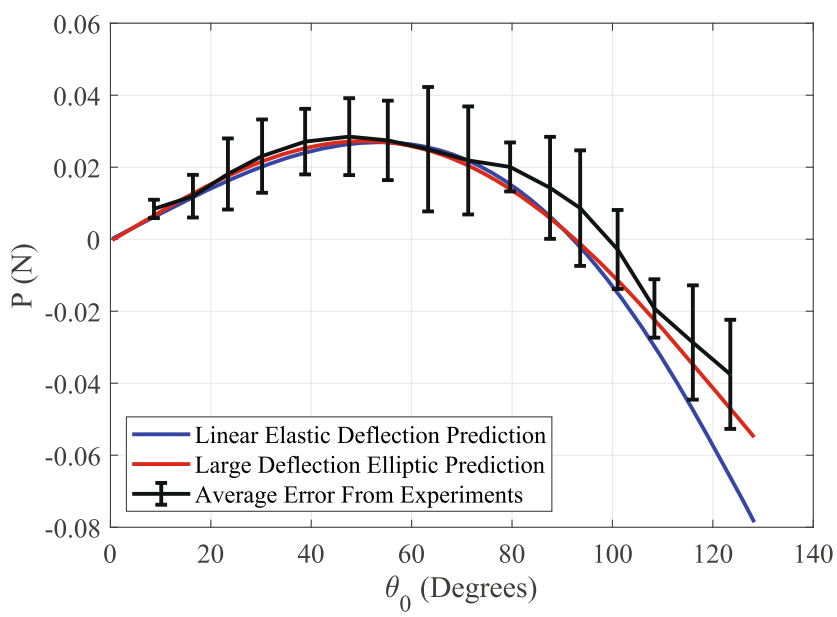

Fig. 9 Compliant joint deflection angle $\left(\theta_{0}\right)$ versus the vertical end load $(P)$ : experimental average error (black), kinematic analysis of nonlinear large angle deflection elliptic solution (red), and kinematic analysis of linear elastic small deflection solution (blue)

constraint on the horizontal coordinate of the beam for the linear elastic deflection case. For design or actuator selection purposes, one can determine the minimum required motor torque for bending the compliant joint to a certain angle through static equilibrium balance for link $A E$ :

$\sum M_{A} \Rightarrow T_{\text {in }}=L_{A E} F_{B} \cos \left(\theta_{0}-\psi\right)$.

Figure 9 shows the simulation and average experimental data for the compliant joint deflection versus vertical force. The error bars on the experimental results, at each deflection amount, represent the standard deviation of six trials. The simulation results are obtained by solving the system of Eqs. (16) to (23) for the linear case, and the nonlinear case is solved by using Eqs. (16) to (26) with the help of elliptic integral solutions. Ascan be seen, the large deflection model predictions match closely with the experimental results over the entire range of simulation, while the linear elastic theory starts deviating more after $90^{\circ}$. The linear formulation cannot accurately predict the behavior of the joint for large deflections since the beam evidently elongates as its length is free to

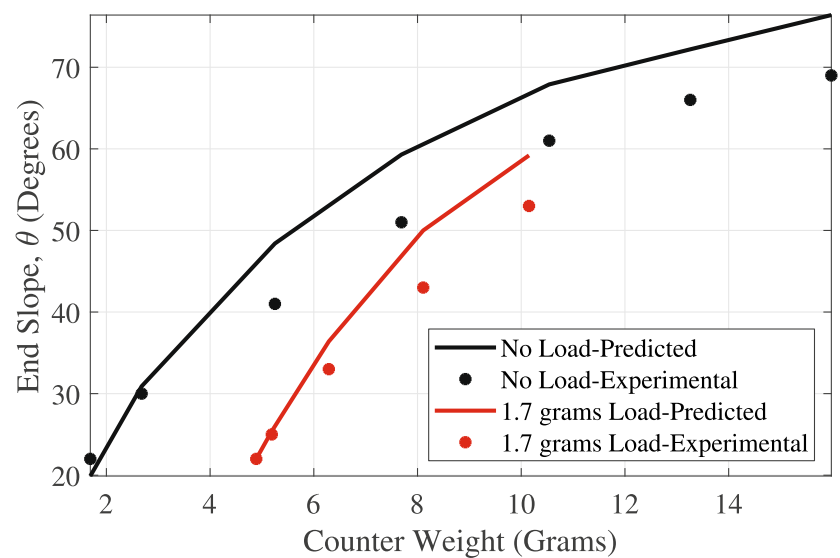

Fig. 11 Experimental torque limit versus angular deflection for an unloaded and loaded leg under a payload of $D y=1.7 \mathrm{~g}$, carried at the tip of the leg (see Figs. 7(b) and 10(b))

change. Thus, the nonlinear theory must be favored for modeling of compliant mechanisms in miniature robots, where the loads are rather small butthe deflections can be as large as $120^{\circ}$. The underestimation of the joint deflection angles in the simulations, compared to the experiments, can be attributed to the friction at the sliding joint and the moment acting on the force sensor due to the position offset between the sensor and the flexure joint. These experiments are run to verify the nonlinear large angle deflection model that is developed in MATLAB. The agreement between the experiments and the simulations suggests that the model is indeed correctly implemented and that it can be developed further to predict the kinematics of MinIAQ's compliant leg mechanism.

\subsection{Payload Capacity and Calculation of Required Motor Torque Using Nonlinear Large Deflection Model}

One of the most important parameters that needs to be investigated during foldable robot design is the maximum motor torque output to decide if an actuator has enough torque to generate leg motion. This output should be higher than the
Fig. 10 Limiting input crank torque experiment setup. (a) No load attached on the tip of the leg. (b) Vertical load attached on the tip of the leg

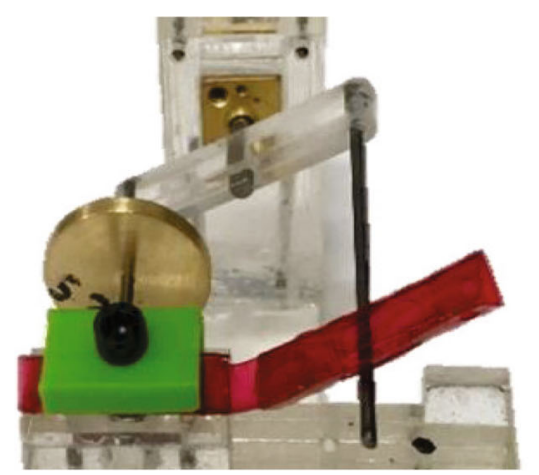

(a)

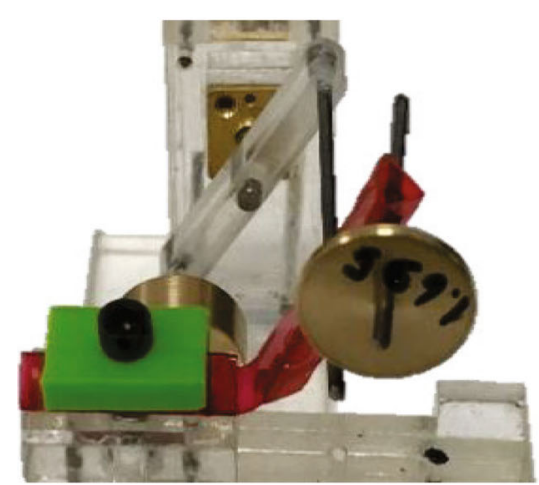

(b) 


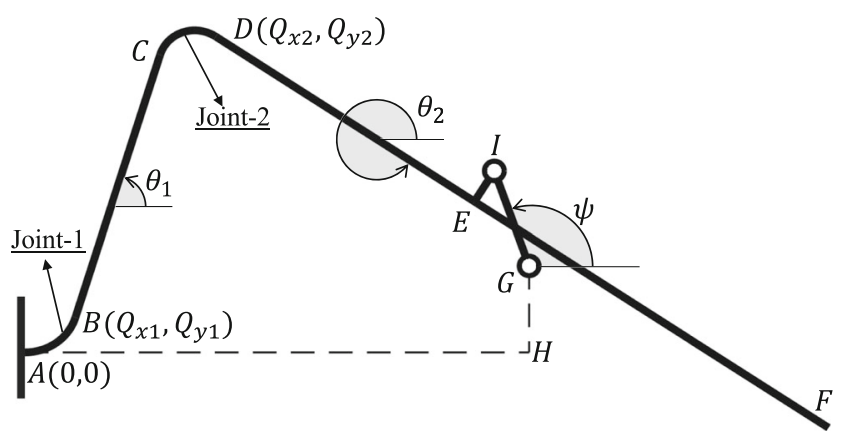

Fig. 12 Compliant leg mechanism of MinIAQ

torque needed for actuation of the locomotion mechanism under robot's weight and the large deflections sustained by the flexure joints. A test setup, shown in Fig. 10, is designed to check the required motor torque for unloaded (when the robot's legs are in air) and vertically loaded (when the robot's legs are touching the ground) cases.

In the setup, instead of a motor, a counterweight is used to create a known torque on input crank. The output of the setup is the end slope of the flexure joint, which is found by measuring the angular displacement of the rigid link on the right side of the flexure joint. The measurements are done using the images captured during the experiments. The rigid link on the left side of the joint is the mechanical ground. An unloaded experiment is shown in Fig. 10(a). External vertical load, which simulates therobot weight, can be added to the setup, as can be seen in Fig. 10(b). The results of the unloaded experiments where the leg is under no external load as well as the results of the loaded experiments where the leg is under an external weight are shown in Fig. 11. Note that the analysis of the test setup mechanism is very similar to that of Section 1 ,

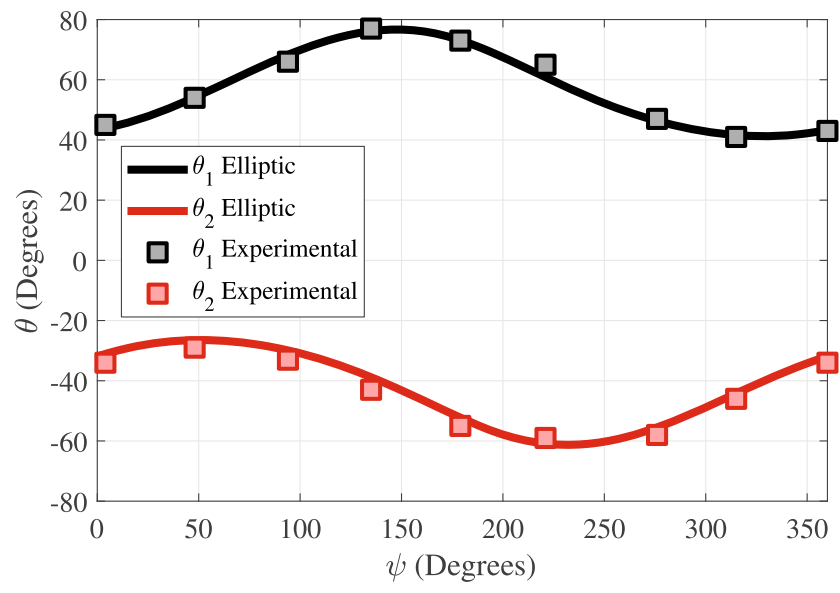

Fig. 14 Experimental and simulation results of MinIAQ's link angles $\left(\theta_{1}\right.$ and $\theta_{2}$ ), obtained by kinematic analysis of the compliant mechanism with large deflection beam theory and elliptic integral solution

and thus is not presented for the sake of brevity. The only differences are that the input to the simulation is the known counterweight torque $T_{i n}$, rather than the crank angle $\psi$, and the vertical load $D_{y}$ is nonzero for the loaded case.

The results show good trend agreement between the simulations and the experiments, where the simulations are estimating more rotation will occur under same motor torque (or less torque is needed for the same rotation amount) compared to the experiments. This difference can be attributed to frictional losses in the system, which are not modeled. As a side note, the simulations, when used with a safety factor, are quite accurate in determining the minimum motor torque required to actuate a mechanism under robot weight, which is a very important parameter during the robot design.
Fig. 13 Free body diagrams of (a) the compliant joint $A B$, (b) the compliant joint $C D$, (c) link $G I$ : the crank link connected to the motor, (d) link $B C$, and (e) link $D F$

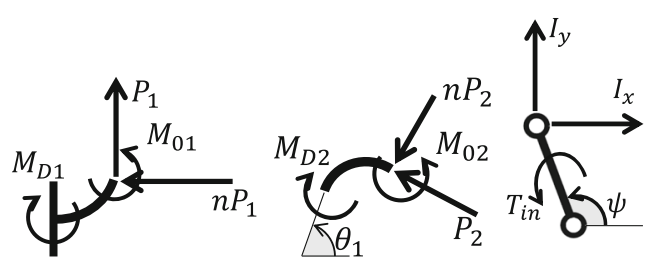

(a)

(b)

(c)

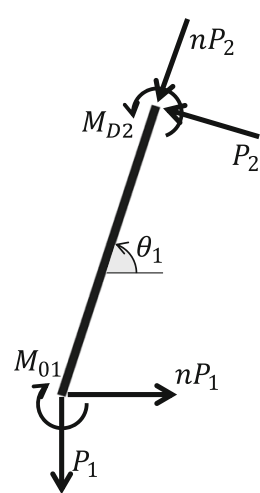

(d)

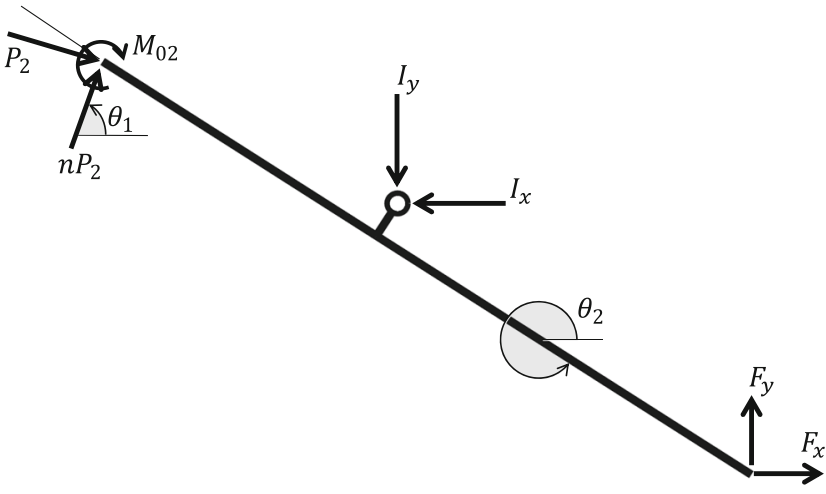

(e) 
Fig. 15 (a) The compliant leg mechanism of MinIAQ showing the experimental foot trajectory obtained by tracking a hole at the tip of the leg via image

processing. (b) Trajectories of the proper compliant leg kinematics (Fig. 3(a)), the simplified pin-joint fourbar kinematics (Fig. 3(b)), and the actual experimental leg setup

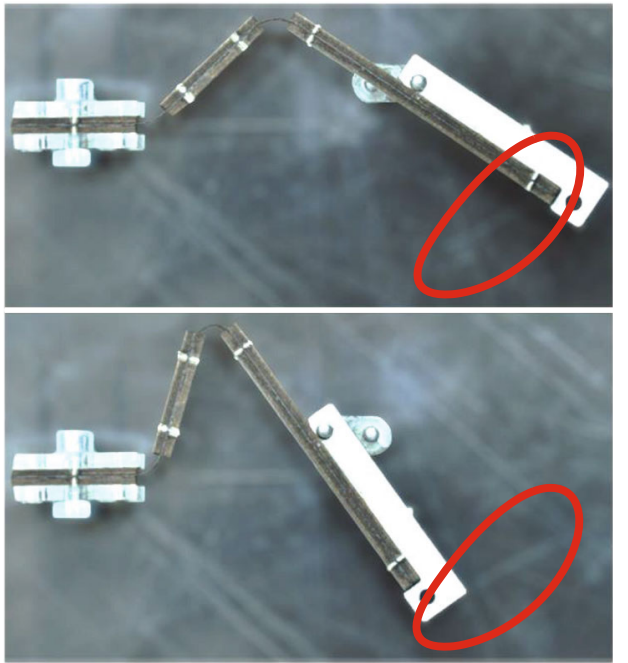

(a)

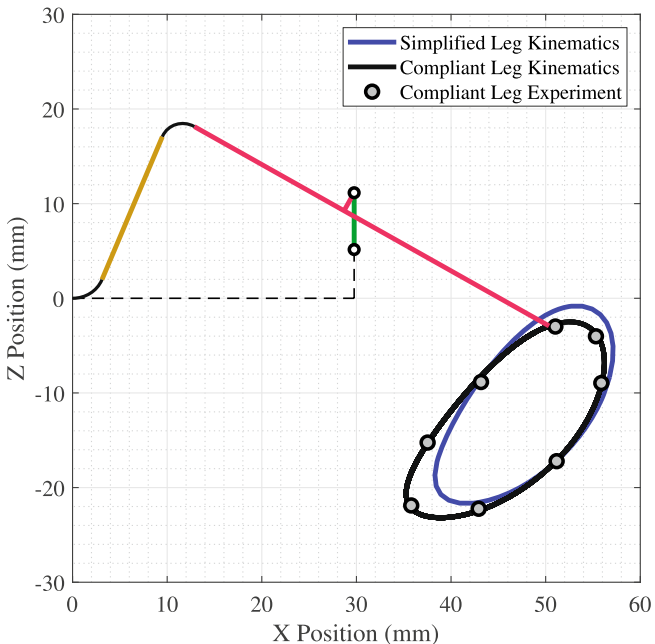

(b)

\subsection{Leg Kinematics of MinIAQ}

The methodology developed in section 1 can be extended for the analysis of MinIAQ's leg mechanism. Accordingly, kinematic and dynamic simulations of MinIAQ are run with a compliant leg model and these results are compared with the results acquired from a simplified rigid-link model. The kinematic model of MinIAQ's compliant mechanism is developed based on the skeleton diagram shown in Fig. 12. In the mechanism, $A B$ and $C D$ are compliant joints and points $G$ and $I$ are ideal revolute/pin joints. $B C, D E, E F, E I$, and $G I$ are rigid links. The motor is positioned at the fixed point $G$, and $\psi$ is the input angle of the crank. Point $F$ is the tip of the leg to which any external load $\left(F_{x}, F_{y}\right)$ may be applied during walking due to friction or contact. The link lengths are denoted by $L_{B C}, L_{D E}, L_{E F}, L_{E I}, L_{E G}, L_{G I}, L_{A H}$ and $L_{G H}$.

For any input crank angle $\psi$, the position kinematics can be expressed by the following loop closure equations:

$Q_{x 1}+L_{B C} \cos \theta_{1}+Q_{x 2}+L_{D E} \cos \theta_{2}+L_{E I} \cos \left(\theta_{2}+\frac{\pi}{2}\right)$
$-L_{G I} \cos \psi-L_{A H}=0$,

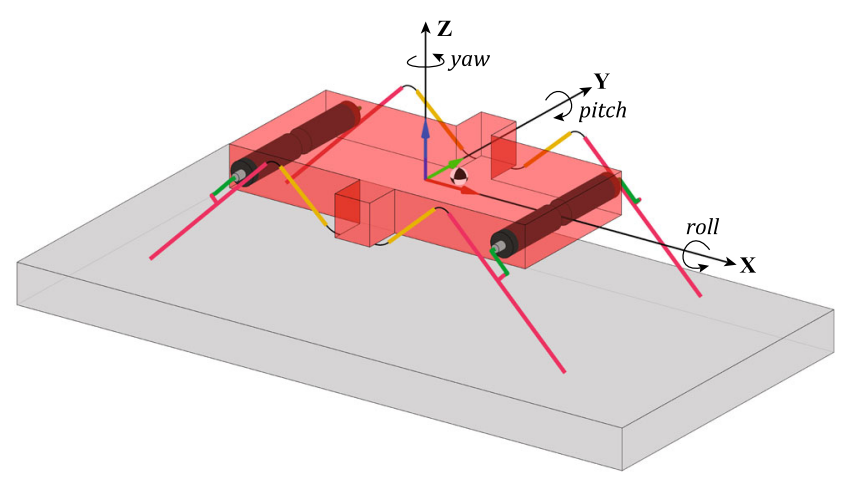

Fig. 16 A representation of the robot's body-attached reference frame used in modeling the dynamics
$Q_{y 1}+L_{B C} \sin \theta_{1}+Q_{y 2}+L_{D E} \sin \theta_{2}+L_{E I} \sin \left(\theta_{2}+\frac{\pi}{2}\right)$

$-L_{G I} \sin \psi-L_{G H}=0$.

The unknown variables $\left(\theta_{1}, Q_{x 1}, Q_{y 1}, \theta_{2}, Q_{x 2}, Q_{y 2}\right)$ in Eqs. (28) and (29) are dependent on the applied loads at the tip of the leg, which are required for determination of the bending amounts of the flexure joints. In this regard, static equilibrium analysis must be done to determine joint deflections and the unknown link angles. This requires drawing the free body diagrams of the links as shown in Fig. 13.

The static equilibrium equations for link $B C$ can be written as:

$\sum F_{x} \Rightarrow-n P_{2} \cos \theta_{1}-P_{2} \sin \theta_{1}+n P_{1}=0$,

$\sum F_{y} \Rightarrow-n P_{2} \sin \theta_{1}+P_{2} \cos \theta_{1}-P_{1}=0$,

$\sum M_{B} \Rightarrow-M_{01}+M_{D 2}+L_{B C} P_{2}=0$,

for link $D F$ as:

$\sum F_{x} \Rightarrow F_{x}-I_{x}+n P_{2} \sin \left(\frac{\pi}{2}-\theta_{1}\right)+P_{2} \cos \left(\frac{\pi}{2}-\theta_{1}\right)=0$,

$\sum F_{y} \Rightarrow F_{y}-I_{y}+n P_{2} \cos \left(\frac{\pi}{2}-\theta_{1}\right)-P_{2} \sin \left(\frac{\pi}{2}-\theta_{1}\right)=0$,

$$
\begin{aligned}
\sum M_{D} \Rightarrow-M_{02} & +L_{D F}\left[F_{y} \cos \left(-\theta_{2}\right)+F_{x} \sin \left(-\theta_{2}\right)\right] \\
& -L_{D E}\left[I_{y} \cos \left(-\theta_{2}\right)+I_{x} \sin \left(-\theta_{2}\right)\right] \\
& -L_{E I}\left[I_{x} \cos \left(-\theta_{2}\right)-I_{y} \sin \left(-\theta_{2}\right)\right]=0
\end{aligned}
$$

and for the compliant joint $\mathrm{CD}$ as:

$\sum M_{C} \Rightarrow-M_{D 2}+M_{02}+Q_{x 2} P_{2}+Q_{y 2} n P_{2}=0$. 
In addition, one can determine the minimum required input torque for the motor using the following relation:

$\sum M_{G} \Rightarrow T_{i n}=L_{G I}\left[I_{x} \sin \psi-I_{y} \cos \psi\right]$.

The inputs to the system of Eqs. 28 to 38 (nine equations) are $\psi, F_{x}$, and $F_{y}$ and the unknown variables are: $\theta_{1}, Q_{x 1}, Q_{y 1}$, $\theta_{2}, Q_{x 2}, Q_{y 2}, I_{x}, I_{y}, P_{1}, n P_{1}, M_{01}, P_{2}, n P_{2}, M_{02}$, and $M_{D 2}$. To solve for these 15 unknowns, at least six more equations are needed, which are obtained by modeling each compliant joint with the nonlinear large deflection theory (similar to Eq. 24, 25 and 26). Solving simultaneously for the static force balance, the large angle deflection model, and the loop closure equations yields the kinematic results. The solution to the developed model is first verified using experimental data.

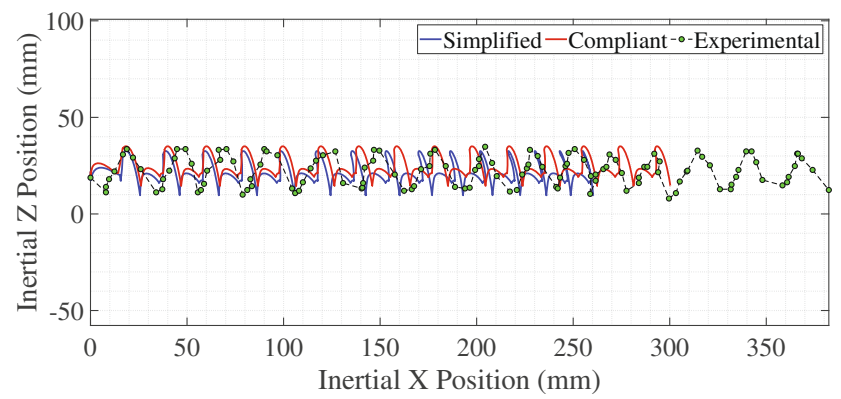

(a)

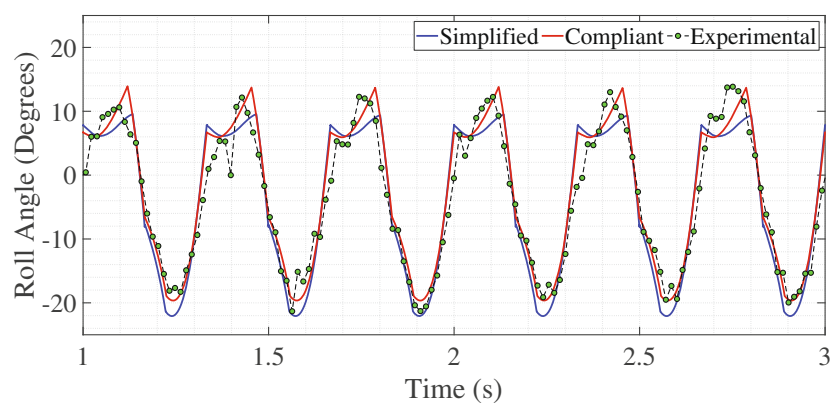

(b)

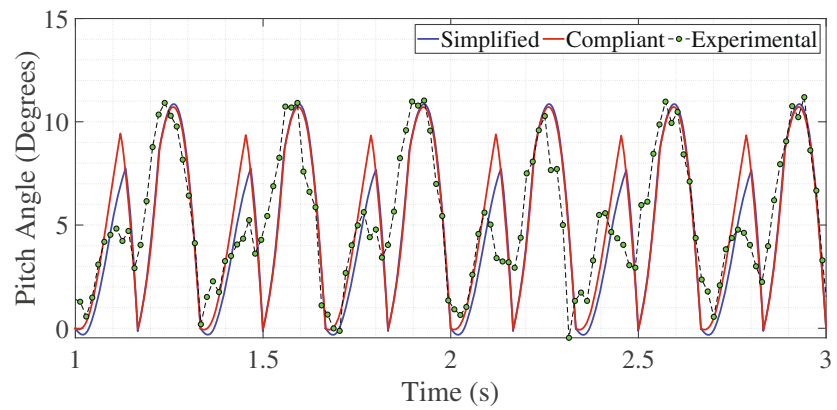

(c)

Fig. 17 Dynamic simulation and experimental results of MinIAQ's trot gait locomotion at $3.0 \mathrm{~Hz}$ motor speed with the compliant and simplified leg kinematics. (a) The side view centroid position. (b) The roll angle comparison. (c) The pitch angle comparison
Simulation is done for a full rotation of the thecrank, in the absence of any contact force at the foot, and the resulting deflections of the flexure joints and the overall mechanism configuration are obtained. Figure 14 represents the variation of $\theta_{1}$ and $\theta_{2}$ link angles and verifies that the kinematic results of the compliant leg model are in good agreement with the experiments. Note that $\theta_{1}$ also represents the bending angle of the flexure joint $A B$, whereas the bending angle of joint $C D$ can be measured as the difference of $\theta_{1}$ and $\theta_{2}$, which exceeds $115^{\circ}$ of deflection at certain instances during a walking cycle. Estimation of compliant joint deflections is of utmost importance during robot design from a fatigue failure perspective. A similar kinematic solution was obtained by FEA method matching very well with the large deflection model, however, the runtime of the FEA method was more than 100 times slower. This implies that the large angle model must be favored over FEA for dynamic simulation of the robot, which is heavily dependent on the leg kinematics.

To further investigate the compliant model advantages, MinIAQ's foot trajectory for two different kinematic models (presented in Fig. 3) are plotted and compared with experimental results. The experimental trajectory path is obtained by using the test setup shown in Fig. 15(a). The kinematic analysis of the simplified rigid-link pin-joint leg mechanism is relatively simple and straightforward; however, it can be clearly seen from Fig. 15(b) that this method has lower accuracy, especially as the flexures get longer. On the other hand, while compliant analysis requires more time and computational power to converge to a solution, the output trajectory agrees very well with the experimental results. Therefore, compliant kinematics using large deflection beam theory and elliptic integral solution can compensate for the higher runtime with satisfactory results.

The major design challenge in miniature foldable robots is designing the motion mechanism trajectory appropriately to achieve a desired path. With the methods outlined here, the trajectory of the compliant motion mechanism can be acquired with a very highaccuracy. The obvious trade-off is the modeling effort spent and the accuracy of the results. If one can accept lower accuracy kinematic modeling, the large deflection based kinematic models are not needed. However, from our experience, this is not always the case; for a miniature foldable robot to move properly, the trajectory of the motion mechanism should be known and optimized. Hence, large angle deflection based kinematic modeling should be performed.

\section{Dynamic Analysis with Different Kinematic Models}

The importance of obtaining an accurate kinematic solution for modeling and designing purposes can be further investigated by analyzing the dynamic behavior of MinIAQ. For the simulation of the robot's locomotion, a 3D rigid body 
dynamic model is developed in MATLAB [15]. A state vector of six translational and six rotational variables is obtained at very small time steps by solving the Newton-Euler equations for five seconds of simulation under trot gait at $3.0 \mathrm{~Hz}$ motor speed. Figure 16 shows a schematic of MinIAQ with the compliant leg model. The body-attached axes $(\mathcal{B})$ placed at the geometric centroid of the robot's frame are used to define the orientation based on Euler angle rotations (roll $\phi$, pitch $\theta$, and yaw $\psi$ ). The position of the body centroid is also tracked over time with respect to an inertial reference frame $(\mathcal{I})$ fixed to the ground level and initially parallel with the body-attached frame (not shown on the figure). The center of mass as well as the inertia tensor were estimated from an actual assembled robot by measuring the position and mass of each individual component of the robot. Notice that the slight shift of the center of mass with respect to the geometric centroid (check the center of gravity, COG, symbol in Fig. 16) causes an imbalance in the body and results in an asymmetric dynamic behavior.

The governing rigid body dynamic equations are given by the following 12 ordinary differential equations:

$\dot{\mathrm{X}}=\left(\begin{array}{c}\dot{P}_{\mathcal{I} x} \\ \dot{P}_{\mathcal{I} y} \\ \dot{P}_{\mathcal{I} z} \\ \dot{\phi} \\ \dot{\theta} \\ \dot{\psi} \\ \dot{u} \\ \dot{v} \\ \dot{w} \\ \dot{p} \\ \dot{q} \\ \dot{r}\end{array}\right)=\left(\begin{array}{l}(\mathrm{c} \theta \mathrm{c} \psi) u+(\mathrm{s} \theta \mathrm{s} \phi \mathrm{c} \psi-\mathrm{s} \psi \mathrm{c} \phi) v+(\mathrm{s} \theta \mathrm{c} \phi \mathrm{c} \psi+\mathrm{s} \psi \mathrm{s} \phi) w \\ (\mathrm{c} \theta \mathrm{s} \psi) u+(\mathrm{s} \theta \mathrm{s} \phi \mathrm{s} \psi+\mathrm{c} \psi \mathrm{c} \phi) v+(\mathrm{s} \theta \mathrm{c} \phi \mathrm{s} \psi-\mathrm{c} \psi \mathrm{s} \phi) w \\ (-\mathrm{s} \theta) u+(\mathrm{c} \theta \mathrm{s} \phi) v+(\mathrm{c} \theta \mathrm{c} \phi) w \\ p+(q \sin \phi+r \cos \phi) \tan \theta \\ q \cos \phi-r \sin \phi \\ \frac{q \sin \phi+r \cos \phi}{\cos \theta} \\ \frac{F_{\mathcal{B} x}-(q w-r v)}{m} \\ \frac{F_{\mathcal{B} y}-(r u-p w)}{m} \\ \frac{F_{\mathcal{B} z}-(p v-q u)}{m} \\ \frac{M_{\mathcal{B} x}-\left(I_{z z}-I_{y y}\right) q r}{I_{x x}} \\ \frac{M_{\mathcal{B} y}-\left(I_{x x}-I_{z z}\right) r p}{I_{y y}} \\ \frac{M_{\mathcal{B} z}-\left(I_{y y}-I_{x x}\right) p q}{I_{z z}}\end{array}\right)$

where $\quad \mathbf{P}_{\mathcal{I}}=\left[\begin{array}{lll}P_{\mathcal{I} x} & P_{\mathcal{I} y} & P_{\mathcal{I}_{z}}\end{array}\right]^{\top}, \quad \Gamma=\left[\begin{array}{lll}\phi & \theta & \psi\end{array}\right]^{\top}$, $\mathrm{V}_{\mathcal{B}}=\left[\begin{array}{lll}u & v & w\end{array}\right]^{\top}$, and $\Omega_{\mathcal{B}}=\left[\begin{array}{lll}p & q & r\end{array}\right]^{\top}$ define the position, orientation, translational speed, and rotational speed of the robot, respectively. Note that $\mathrm{c}$ and $\mathrm{s}$ are abbreviations for cosine and sine functions, respectively, $m$ denotes the mass of the robot, and $\left\{I_{x x}, I_{y y}, I_{z z}\right\}$ represent the mass moments of inertia of the robot's rigid body about the body-attached axes. The inputs to the system are the net external force and moment $\left(\mathrm{F}_{\mathcal{B}}\right.$ and $\left.\mathrm{M}_{\mathcal{B}}\right)$ along the body-attached axes, that are determined by a nonlinear viscoelastic spring-damper model based on feet-ground interactions. The contact force model is an implicit function of all system states as well as the leg kinematics. Detailed derivation of the dynamics and the contact force model are presented in [15].

Two different sets of simulation are run in which all the dynamic parameters are held the same except for switching the leg kinematic model between the compliant model and the simplified fourbar (see Fig. 3). Since MinIAQ is originally designed for trot gait, most experiments are run with trot gait straight walk at 3.0 Hz motor frequency and experimental data are extracted from video footage for comparison with the simulations. Note that the robot has no on-board sensing device (gyroscope or accelerometer) to record the position and orientation data and thus, this is done using raw video shots of multiple runs. The position of the centroid and variations of pitch angle are measured from the side view shots, and the roll angle is measured from the frontal shots.

MinIAQ's dynamic simulation and experimental results are shown in Fig. 17. Note that Fig. 17(a) represents the global centroid position of the robot for the full five second period of simulation; whereas, the pitch and roll angle results (Figs. 17(b) and 17(c)) are cut down to two seconds of simulation, due to their periodicity, for better clarity. As can be seen, neither the compliant mechanism kinematics nor the simplified pin-joint fourbar model can exactly predict the position and speed of the robot in space. It should be noted here that predicting a miniature robot's dynamics is often relatively harder compared to predicting the dynamics of a macro scale one because there are more sources of error and uncertainty in experiments. This can be clearly seen from the change in average robot speed between different steps in the experiments, which ideally should not happen. The slip-stiction problem of small legs, poor estimation of friction and impact parameters, low speed controller response, and lack of proper on-board sensors are some of the issues to which the mismatching can be attributed to. On the other hand, the simulations with the compliant leg goes along a much more straight path and in a stable manner compared to the simulations with the simplified mechanism where the robot starts to turn towards its heavier side (shifted COG). This is in-line with our experimental results, where the robot moves along a relatively straight and stable path.

The simulation results of roll and pitch variations show a good trend agreement with the experimental data. The compliant mechanism predicts the rolling effect very well, while the simplified leg model slightly underestimates the actual maximum body rolls. For the pitch angle results, both cases predict the maximum experimental pitch correctly, however they both overestimate the actual pitching during the steps of the feet lift on the heavier side. All in all, the dynamic simulation of the robot with the compliant leg model yields more accurate and stable results than the simplified kinematics at the cost of longer runtime. The simulation runtime can be improved if the kinematic solution is initially pre-solved once for a fine domain of inputs and a lookup 
table is generated for interpolating the kinematics during future dynamic simulation runs.

\section{Conclusions}

Accurate kinematic modeling of miniature foldable robot mechanisms is very important to estimate the motion of the robot. In this work, kinematic modeling of compliant mechanisms has been investigated based on elliptic integral solutions of large deflection model by means of combining the loop closure and static equilibrium equations. In order to check the validity of the compliant kinematic model developed, a simple single flexure joint compliant leg mechanism has been used. The comparison of experimental force data generated by the flexure joint with the simulations has shown that elliptic integral kinematic solution predictions are accurate and the large deflection model is implemented properly. Following that step, kinematic analysis of the compliant leg mechanism used in MinIAQ has been conducted using the same method. With this kinematic model, we can obtain the trajectory of the tip of the leg, maximum load carrying capacity with respect to limiting material properties and the motor torque requiredto rotate the crank link. The main advantage of the large deflection kinematic analysis is that the trajectory of the foot noticeably differs from traditional revolute joint kinematic analysis and is very close to the experimental results. Although it takes longer to converge to a solution compared to linear beam theory or simplified kinematic methods, the compliant mechanism kinematic analysis based on large deflection theory gives a much accurate solution. If the trajectory path is very sensitive for acompliant mechanism application (and it often is the case for miniature foldable robots), it is vital to use elliptic integral solution in the design stage. This large deflection model of MinIAQ legs are then integrated with a rigid body dynamic model ofour foldable miniature robot and the results are compared with a dynamic simulation incorporating a simplified leg model and experimental results. The large deflection leg model predicts the actual robot's dynamic behavior better than the simplified model with the trade-off in modeling complexity and simulation runtime. The results presented here can be used by other foldable robotics researchers to decide if they would like kinematic and dynamic simulations of their robots with lower complexity, faster runtime and lower accuracy or slower, complex, but more accurate results.

As the future work, we would like to focus on fatigue analysis of joints in foldable robotics applications. The large deflections occurring at compliant mechanisms often cause the joints to fail under cyclic load, such as in the actuation of compliant legmechanisms in the foldable robots case. This issue shortens the life of the compliant mechanism, hence the robot's. If fatigue failure analysis can be done on the foldable robot flexure joints during the design phase, we can manufacture longer lasting and thus more impactful foldable miniature robots.

Acknowledgements The authors would like to thank all members of Bilkent Miniature Robotics Laboratory for their invaluable contributions to this work. This project is funded by The Scientific and Technological Research Council of Turkey (TUBITAK) through 3001 program (Grant number: 215 M366).

\section{Compliance with Ethical Standards}

Conflict of Interest The authors declare that they have no conflict of interest.

\section{References}

1. Shimada, E., Thompson, J., Yan, J., Wood, R., Fearing, R.: Prototyping millirobots using dextrous microassembly and folding. Proc ASME IMECE/DSCD. 69(2), 933-940 (2000)

2. Wood, R.J., Avadhanula, S., Sahai, R., Steltz, E., Fearing, R.S.: Microrobot design using fiber reinforced composites. J. Mech. Des. 130(5), 052304 (2008)

3. Whitney, J.P., Sreetharan, P.S., Ma, K.Y., Wood, R.J.: Pop-up book mems. J. Micromech. Microeng. 21(11), 115021 (2011)

4. Onal CD, Wood RJ, Rus D (2011) Towards printable robotics: origami-inspired planar fabrication of three-dimensional mechanisms. In: robotics and automation (ICRA), 2011 IEEE international conference on, IEEE, pp 4608-4613

5. Felton, S., Tolley, M., Demaine, E., Rus, D., Wood, R.: A method for building self-folding machines. Science. 345(6197), 644-646 (2014)

6. Karakadioglu C, Askari M, Ozcan O (2017) Design and operation of MinIAQ: an untethered foldable miniature quadruped with individually actuated legs. In: IEEE international conference on advanced intelligent mechatronics (AIM'17), pp 247-252

7. Mehta AM, Rus D (2014) An end-to-end system for designing mechanical structures for print-and-fold robots. In: 2014 IEEE international conference on robotics and automation (ICRA), IEEE, pp 1460-1465

8. Howell LL (2001) Compliant Mechanisms. John Wiley \& Sons

9. Faal, S.G., Chen, F., Tao, W., Agheli, M., Tasdighikalat, S., Onal, C.D.: Hierarchical kinematic design of foldable hexapedal locomotion platforms. Journal of Mechanisms and Robotics. 8(1), 011005 (2016)

10. Karydis K, Poulakakis I, Tanner HG (2012) A switching kinematic model for an octapedal robot. In: 2012 IEEE/RSJ international conference on intelligent robots and systems, IEEE, pp 507-512

11. Ozcan O, Baisch AT, Ithier D, Wood RJ (2014) Powertrain selection for a biologically-inspired miniature quadruped robot. In: robotics and automation (ICRA), 2014 IEEE international conference on, IEEE, pp 2398-2405

12. Soltero DE, Julian BJ, Onal CD, Rus D (2013) A lightweight modular 12-dof print-and-fold hexapod. In: 2013 IEEE/RSJ international conference on intelligent robots and systems, IEEE, pp 14651471

13. Finio, B.M., Wood, R.J.: Distributed power and control actuation in the thoracic mechanics of a robotic insect. Bioinspiration \& biomimetics. 5(4), 045006 (2010)

14. Hoover AM, Fearing RS (2009) Analysis of off-axis performance of compliant mechanisms with applications to mobile millirobot design. In: 2009 IEEE/RSJ international conference on intelligent robots and systems, IEEE, pp 2770-2776 
15. Askari M, Ozcan O (2019) Dynamic modeling and gait analysis for miniature robots in the absence of foot placement control. In: IEEE international conference on robotics and automation (ICRA'19), pp 9754-9760

16. Hoffman, K.L., Wood, R.J.: Myriapod-like ambulation of a segmented microrobot. Auton. Robot. 31(1), 103 (2011)

17. Saranli, U., Buehler, M., Koditschek, D.E.: Rhex: a simple and highly mobile hexapod robot. The International Journal of Robotics Research. 20(7), 616-631 (2001)

18. Shigley JE (2011) Shigley's Mechanical Engineering Design. Tata McGraw-Hill Education

19. Banerjee, A., Bhattacharya, B., Mallik, A.: Large deflection of cantilever beams with geometric non-linearity: analytical and numerical approaches. International Journal of Non-Linear Mechanics. 43(5), 366-376 (2008)

20. Bisshopp, K., Drucker, D.: Large deflection of cantilever beams. Q. Appl. Math. 3(3), 272-275 (1945)

21. Holst, G.L., Teichert, G.H., Jensen, B.D.: Modeling and experiments of buckling modes and deflection of fixed-guided beams in compliant mechanisms. J. Mech. Des. 133(5), 051002 (2011)

22. Howell, L.L., Leonard, J.N.: Optimal loading conditions for nonlinear deflections. International journal of non-linear mechanics. 32(3), 505-514 (1997)

23. Howell, L.L., Midha, A.: Parametric deflection approximations for end-loaded, large-deflection beams in compliant mechanisms. J. Mech. Des. 117(1), 156-165 (1995)

24. Kimball, C., Tsai, L.W.: Modeling of flexural beams subjected to arbitrary end loads. J. Mech. Des. 124(2), 223-235 (2002)

25. Mattiasson, K.: Numerical results from large deflection beam and frame problems analysed by means of elliptic integrals. Int. J. Numer. Methods Eng. 17(1), 145-153 (1981)

26. Saxena, A., Kramer, S.: A simple and accurate method for determining large deflections in compliant mechanisms subjected to end forces and moments. J. Mech. Des. 120(3), 392-400 (1998)

27. Shoup, T.E., McLarnan, C.W.: On the use of the undulating elastica for the analysis of flexible link mechanisms. Journal of Engineering for Industry. 93(1), 263-267 (1971)

28. Zhang, A., Chen, G.: A comprehensive elliptic integral solution to the large deflection problems of thin beams in compliant mechanisms. Journal of Mechanisms and Robotics. 5(2), 021006 (2013)

29. Chen, G., Xiong, B., Huang, X.: Finding the optimal characteristic parameters for 3r pseudo-rigid-body model using an improved particle swarm optimizer. Precis. Eng. 35(3), 505-511 (2011)

30. Mattson, C.A., Howell, L.L., Magleby, S.P.: Development of commercially viable compliant mechanisms using the pseudo-rigidbody model: case studies of parallel mechanisms. J. Intell. Mater. Syst. Struct. 15(3), 195-202 (2004)

31. Midha, A., Bapat, S.G., Mavanthoor, A., Chinta, V.: Analysis of a fixed-guided compliant beam with an inflection point using the pseudo-rigid-body model concept. Journal of Mechanisms and Robotics. 7(3), 031007 (2015)

32. Ramirez IA (2014) Pseudo-Rigid-Body Models for Approximating Spatial Compliant Mechanisms of Rectangular Cross Section. University of South Florida

33. Su HJ (2008) A load independent pseudo-rigid-body $3 \mathrm{r}$ model for determining large deflection of beams in compliant mechanisms. Proceedings of ASME IDETC/CIE (43260) pp 109-121
34. Su, H.J.: A pseudorigid-body $3 \mathrm{r}$ model for determining large deflection of cantilever beams subject to tip loads. Journal of Mechanisms and Robotics. 1(2), 021008 (2009)

35. Venkiteswaran, V.K., Su, H.J.: A three-spring pseudorigid-body model for soft joints with significant elongation effects. Journal of Mechanisms and Robotics. 8(6), 061001 (2016)

36. Yu, Y.Q., Feng, Z.L., Xu, Q.P.: A pseudo-rigid-body 2r model of flexural beam in compliant mechanisms. Mech. Mach. Theory. 55, 18-33 (2012)

37. Yu, Y.Q., Zhu, S.K., Xu, Q.P., Zhou, P.: A novel model of large deflection beams with combined end loads in compliant mechanisms. Precis. Eng. 43, 395-405 (2016)

38. Chase Jr., R.P., Todd, R.H., Howell, L.L., Magleby, S.P.: A 3-d chain algorithm with pseudo-rigid-body model elements. Mechanics Based Design of Structures and Machines. 39(1), 142 156 (2011)

39. Hill, T., Midha, A.: A graphical, user-driven newton-raphson technique for use in the analysis and design of compliant mechanisms. J. Mech. Des. 112(1), 123-130 (1990)

40. Miller, R.: Numerical analysis of a generalized plane elastica. Int. J. Numer. Methods Eng. 15(3), 325-332 (1980)

41. Tolou, N., Herder, J.: A seminalytical approach to large deflections in compliant beams under point load. Math. Probl. Eng. (2009)

42. Campanile, L., Hasse, A.: A simple and effective solution of the elastica problem. Proc. Inst. Mech. Eng. C J. Mech. Eng. Sci. 222(12), 2513-2516 (2008)

43. Frisch-Fay, R.: Applications of approximate expressions for complete elliptic integrals. Int. J. Mech. Sci. 5(3), 231-235 (1963)

Cem Karakadıoğlu is a Mechanical Engineering graduate, having reveived his B.S. (2015) at Gazi University and his M.S. (2018) at Bilkent University in Ankara, Turkey. During his graduate studies, he conducted research on miniature foldable quadruped robots under supervision of Dr. Onur Özcan at Bilkent Miniature Robotics Laboratory. He is the first alumni of Bilkent Miniature Robotics Laboratory and since 2018, he has been working at Roketsan Inc. as an R\&D engineer.

Mohammad Askari received the B.S. (2015) and M.S. (2018) degrees, both with highest distinction, in mechanical engineering from Middle East Technical University and Bilkent University in Ankara, Turkey, respectively. He is currently a research assistant at Bilkent University and has been with the Miniature Robotics Laboratory since 2015. His current research interests include control, dynamic modeling, simulation, and gait analysis of miniature mobile robots.

Onur Ozcan received his B.S. (2007) in Mechatronics Engineering at Sabanci University in Istanbul, Turkey and his M.S. (2010) and Ph.D. (2012) in Mechanical Engineering at Carnegie Mellon University in Pittsburgh, Pennsylvania. Following PhD, he joined Harvard University Microrobotics Laboratory as a postdoctoral researcher working on miniature crawling robots. Since 2015, he has been an assistant professor at Bilkent University Mechanical Engineering Department in Ankara, Turkey and he has been leading the Bilkent Miniature Robotics Laboratory. 